\title{
Joint Special Issue on PHM for Aerospace Systems
}

\section{Letter from the Guest Editors}

\section{Chetan Kulkarni ${ }^{1}$ and Kai Goebel ${ }^{2}$}

\author{
${ }^{1}$ Senior Scientist, KBR Inc., NASA Ames Research Center, USA \\ ${ }^{2}$ Lab Director, Intelligent Systems Lab,, Palo Alto Research Center, USA
}

Prognostics and health management (PHM) is becoming one of the most popular topics for research and development in the aviation industry. The reasons for this are varied, but one of the main ones is that PHM affords the operator with a way to reduce lifecycle operating costs without necessarily adding expensive accessories that might need to be certified. Many of the papers in this special issue discuss PHM techniques that are based on post flight, or off-board, data processing that adds benefits without additional regulatory constraints. On the other hand, regulators themselves are keenly looking at changing regulations to allow more PHM equipment on board and to allow suppliers and operators to gain maintenance credits from their use. All in all, it is a exciting time to be a engineering working on these topics in the aviation sector. While progress is being made in developing better sensors, models, and analytical methods, the field of aviation itself is changing rapidly. It is seeking to become more sustainable by increasing propulsion efficiencies, it has many players looking to develop and commercialize electrical systems, and it is fast undertaking a digital transformation of the entire ecosystem. In response, system developers and researchers in the field are working on a number of key technologies and methodologies to solve some of the issues that these changes have wrought. This special issue on PHM for Aerospace Systems provides a forum to discuss recent advances pertaining to papers that address topics in this field with an emphasis on Prognostics and Health Management.

These topics include advances in anomaly detection and diagnostics, uncertainty management for prognostics, resilient design for fault-tolerant operations, systematic evaluation of PHM for maintenance processes, assessment of retrofit solutions for particular aircraft systems, PHM informed decision-making for safe operations of autonomous aerial systems, impact of noise on prognostic performance, and lessons learned from trends in aircraft fatigue failure accidents for Structural Health Monitoring.

In the paper by Afshin et al, the authors discuss moment gyros, which are known for their applications in attitude stabilization for aerospace application. These actuators are susceptible to malfunction, which results in faults and failures. Fault detection can improve the reliability of completing a mission while reducing maintenance costs. In this work a model-based fault diagnosis method is proposed. A condensed approach to alleviate the delay caused by the filter's confidence in its estimations is being implemented. To evaluate the performance, a case-study on a closed-loop controlled satellite is provided along with an extensive Monte Carlo simulation. The results provide evidence that the enhanced method can achieve superior performance while requiring less computational resources by eliminating extra grid search loops. 
Next, authors Kyprianidis, et al., discuss the use of advanced engine health monitoring and diagnostic systems which greatly benefit users by helping them avoid potentially expensive and time-consuming repairs by proactively identifying shifts in engine performance trends and make optimal maintenance decisions. Engine health deterioration can manifest itself in terms of rapid/abrupt and gradual performance deviations. An effective engine life-cycle monitoring and diagnostic system is therefore required to be capable of discriminating these two deterioration mechanisms followed by isolating and estimating the rapid fault, with consistent accuracy. In the proposed solution, this diagnostic problem is addressed through a combination of adaptive gas path analysis (AGPA) and artificial neural network (ANN) technique. The performance of the proposed method has been demonstrated and validated based on data obtained from a triple-shaft turbofan engine. The effectiveness of the combined approach has also been compared with the AGPA alone and the advantages and shortcomings highlighted.

In the third paper, Meissner, et al., describe how the aviation industry is continuously introducing digital technologies to automate the state detection of their assets and derive maintenance decisions so as to reduce operating costs and increase operational stability. Many industry efforts and research activities have focused on an early state fault detection and the prediction of system failures. Since research has mainly been limited to the calculation of remaining useful lifetimes (RUL) and has neglected the impact on surrounding processes, changes on the objectives of the involved stakeholders, resulting from these technologies, have hardly been addressed in existing work. In this work, the authors present key features of an automatic condition monitoring architecture for the example of a Tire Pressure Indication System (TPIS). Furthermore, they develop a prescriptive maintenance strategy by modeling the involved stakeholders of aircraft and line maintenance operations with their functional dependencies. Subsequently, they estimate the expected implications for a small aircraft fleet with the introduction of such a monitoring system with various levels of technological maturity. Additionally, they calculate the maintenance cost savings potential for different measurement strategies and compare these results to the current state-of-the-art maintenance approach. To estimate the effects of implementing an automated condition monitoring system, they use a discrete-event, agent-based simulation setup with an exemplary flight schedule and a simulated time span of 30 calendar days. The results observed allow a comprehensive estimation of the maintenance related implications on airline operation and provide key aspects in the development of an airline's prescriptive maintenance strategy.

Autin, et al., discuss Electro-Hydraulic Servo-Actuators (EHSA) which are today a widely adopted solution for primary flight control systems of civil and military aircrafts. Although, some alternatives have emerged in the last decade, such as those based upon electro-mechanical or electro-hydrostatic technologies, electro-hydraulic systems are still considered the most effective option in flight-critical application of new commercial aircrafts. Moreover, the vast majority of aircraft currently in service are equipped with this technology; considering the number of actuators typically employed 
in a primary flight control system and the expected service life of a commercial aircraft, the development of an effective PHM system could provide significant benefits to fleet operators and aircraft maintenance. This paper presents the results of a feasibility study of such a system for electro-hydraulic actuators used in fly-by-wire primary flight control systems, considering the actuator of a wide body commercial aircraft as use case. Aim of the research is the implementation of a PHM system without the addition of dedicated sensors, a solution which would allow for the application of the proposed prognostic solution on both new and existing platforms. The work presented, describes the methodology and the results of the feasibility study through simulation and experimental activities, which shows how the novel PHM technologies proposed for a PHM system for the EHSAs of primary flight control actuators can allow the migration from scheduled to on-condition maintenance.

In the next paper, Sridhar, et al., discuss the health monitoring of mechanical transmission systems, an important area of research not just for aerospace systems, but for most of the mobility sector. Mechanical transmission systems especially gear boxes in aircraft, automobiles, and wind turbines etc. account for many of the maintenance costs due to repairs, replacements and downtime. Gear boxes can experience high levels of failure due to varied load conditions and harsh environments. Replacing the gearbox is quite an expensive process and has significant downtime. Current gearbox monitoring involves mainly measuring vibrations, however vibrations occur when the fault in the gear has already progressed significantly. Gear teeth monitoring lacks sensor technology to successfully detect tooth damage and misalignment. In this work the authors present a new concept, gear teeth damage detection using eddy current sensors fitted on to the teeth of an idler gear at various locations. These sensors detect various faults encountered in a gear such as micro and macro pitting of the tooth surface, contact wear etc. Eddy current sensors are already being used to detect turbomachinery blade vibrations and tip clearance as they are robust and immune to contamination. In the present case, the idler gear incorporates miniature eddy current sensors and state of the art electronics with wireless data transmission to enable the device to operate remotely and in harsh environments. A rotating rig with gears (spur and helical) and oil supply was built to test and validate the sensor by seeding various faults on the tooth surface. The results show that the idler sensor gear was able to detect various faults. The new eddy current sensor idler gear concept will enable health monitoring of the gearbox and will ensure timely maintenance and reduction in operation costs.

Corbetta, et al., propose a perspective towards establishing a framework for uncertainty quantification of autonomous aerospace vehicles for tracking and health monitoring. The approach leverages the use of a predictive process structure, which maps uncertainty sources and their interaction according to the quantity of interest and the goal of the predictive estimation. It is systematic and uses basic elements that are system agnostic, and therefore needs to be tailored according to the specificity of the application. This work is motivated by the interest in low-altitude unmanned aerial vehicle operations, where awareness of vehicle and airspace state becomes more relevant as the density of autonomous operations grows rapidly. Predicted scenarios in 
the area of small vehicle operations and urban air mobility have no precedent, and holistic frameworks to perform prognostics and health management (PHM) at the system- and airspace-level are missing formal approaches to account for uncertainty. At the end of the paper, two case studies demonstrate the implementation framework of trajectory tracking and health diagnosis for a small unmanned aerial vehicle.

Next, Quinones, et al., discuss safe operations for low-flying unmanned aerial vehicles (UAVs) in urban spaces. Given the uncertainties in their operational environments that may be caused by wind gusts and risk of collision with static and dynamic objects, it becomes imperative to develop online decision making schemes based on risk analysis to ensure safe flights, and to deploy replanning algorithms and contingency management schemes to overcome unsafe situations. In this paper, they discuss an online decision making framework that uses a path replanning algorithm to maintain safe operations of UAVs in urban scenarios. Online monitoring of the UAV combines the effects of degrading components and environmental conditions to compute a risk value for the current flight trajectory. Along with this analysis, they propose an online re-planning algorithm that adjusts the flight trajectory to reduce the overall risk to acceptable levels, and thereby maintain flight safety. They demonstrate effectiveness of our approach by running simulation experiments in urban scenarios.

Schumann, et al., describe an autonomous unmanned aerial system (UAS) which needs, during the flight, accurate information about the current failure state of the aircraft and its capabilities in order to safely perform its mission and properly react to contingencies. The flight battery of an electric-propulsion aircraft is its most relevant resource. Model-based prognostics algorithms are used to obtain good estimates of its current state of charge and remaining capacity. In this paper, we focus on the role battery prognostics plays for the safe operation of a highly autonomous aircraft: prognostics for (1) continuous on-board safety monitoring, (2) for UAS operations, and (3) for contingency planning. We present the NASA Autonomous Operating System (AOS) and discuss how the autonomous components closely work together with on-board and server-based ground prognostics systems. We will illustrate the system with case studies on small NASA unmanned aircraft.

The manuscript by Hulse, et al., introduce a framework for incorporating resilience in design to ensure the long-term viability of complex engineered systems. Complex aerospace systems, for example, must ensure safety in the event of hazards resulting from part failures and external circumstances while maintaining efficient operations. Traditionally, mitigating hazards in early design has involved experts manually creating hazard analyses in a time-consuming process that hinders one's ability to compare designs. Furthermore, as opposed to reliability-based design, resilience-based design requires using models to determine the dynamic effects of faults to compare recovery schemes. While many theoretical frameworks have been presented for early hazard assessment, most currently-available modelling tools are meant for the later stages of design. Given the wide adoption of Python in the broader research community, there is an opportunity to create an environment for researchers to study the resilience of 
different PHM technologies in the early phases of design. This paper describes fmdtools, an attempt to realize this opportunity with a set of modules which may be used to construct different design models, simulate system behaviors over a set of fault scenarios and analyze the resilience of the resulting simulation results. This approach is demonstrated in the hazard analysis and architecture design of a multi-rotor drone, showing how the toolkit enables a large number of analyses to be performed on a relatively simple model as it progresses through the early design process.

In Nayak, et al., the removal of noise from signals obtained through the health monitoring systems in gas turbines for accurate prognostics is illuminated. Several filters have been designed and tested for this purpose, and their performance analysis has been conducted. Linear filters are inefficient in the removal of outliers and noise because they cause smoothening of the sharp features in the signal which can indicate the onset of a fault event. On the other hand, non-linear filters based on image processing methods can provide more precise results for gas turbine health signals. Among others, the weighted recursive median (WRM) filter has been shown to provide greater accuracy due to its weight adaptability depending on the signal type. However, sampling data at high rates is possible which needs hardware implementation of the filter. In this paper, the design, simulation and implementation of WRM filters on the FPGA (Field Programmable Gate Arrays) platforms Vivado Design Suite by Xilinx and Quartus Pro Lite Edition 19.3 has been performed. The architectural detail and performance result with the FPGA filters when subjected to abrupt and gradual fault signal is presented.

Wild, et al., investigate trends in aircraft fatigue failure accidents to inform aerospace Structural Health Monitoring (SHM) system Research and Development (R\&D). The research involved collecting 139 aircraft fatigue failure accident reports from the Aviation Safety Network database, which were coded using a directed content analysis. The trends and features of the categorical data were then explored using an ex-post facto study. The results showed that fatigue failure accidents have increased at a rate of $(3.4 \pm 0.6) \times 10-2$ per year since the 1920's. Over the period of the study there were 2098 fatalities in 57 fatal accidents, giving $(15.1 \pm 1.6)$ fatalities per accident and a fatal accident percentage of $(45 \pm 10) \%$. While there is a desire to further improve safety for large transport category aircraft, results indicate that smaller aircraft and operators have seen a relative increase in fatigue failure accidents, and hence are also in need of SHM systems. Engine and undercarriage systems have the greatest number of fatigue failure accidents associated with them, suggesting these should be the focus of SHM R\&D.

We hope that this special joint issue of the International Journal of PHM and SAE International Journal of Aerospace will increase the awareness about innovations in the development of PHM technologies in aerospace and believe that this inspires researchers to focus more attention on this important topic. We would like to express our sincere thanks to all the reviewers of this issue who have generously contributed their valuable time and effort to ensure that it lives up to the Journal's high quality. 
We are also sincerely grateful to Dr. Ravi Rajamani, Editor-in-Chief of the SAE International Journal of Aerospace, and Dr. Abhinav Saxena, and Dr. Marcos Orchard Editors-in-Chief at the International Journal of Prognostics and Health Management, for their constant help and encouragement. This special joint issue would not have been possible without the able assistance of the staff of the SAE International Journal of Aerospace, and especially to Jonathan Neu, Kimberly Martin, and Christie Inman for their support in preparing this special issue.

Chetan Kulkarni, Guest Editor Senior Scientist, KBR Inc. NASA Ames Research Center, USA

Kai Goebel, Guest Editor VP, Intelligent Systems Lab, Palo Alto Research Center, USA 